\title{
(Un)true deuterium abundance in the Galactic disk
}

\author{
Tijana Prodanović ${ }^{1}$, Gary Steigman ${ }^{2}$ and Brian D. Fields ${ }^{3}$ \\ ${ }^{1}$ Department of Physics, University of Novi Sad, \\ Trg Dositeja Obradovića 4, 21000 Novi Sad, Serbia \\ email: prodanvc@df.uns.ac.rs \\ ${ }^{2}$ Department of Physics and Astronomy, Ohio State University, \\ 191 W. Woodruff Ave., Columbus OH 43210-1117, USA \\ email: steigman@mps.ohio-state.edu \\ ${ }^{3}$ Department of Astronomy, University of Illinois, \\ 1002 W. Green St., Urbana IL 61801, USA \\ email: bdfields@illinois.edu
}

\begin{abstract}
Deuterium has a special place in cosmology, nuclear astrophysics, and galactic chemical evolution, because of its unique property that it is only created in the big bang nucleosynthesis while all other processes result in its net destruction. For this reason, among other things, deuterium abundance measurements in the interstellar medium (ISM) allow us to determine the fraction of interstellar gas that has been cycled through stars, and set constraints and learn about different Galactic chemical evolution (GCE) models. However, recent indications that deuterium might be preferentially depleted onto dust grains complicate our understanding about the meaning of measured ISM deuterium abundances. For this reason, recent estimates by Linsky et al. (2006) have yielded a lower bound to the "true", undepleted, ISM deuterium abundance that is very close to the primordial abundance, indicating a small deuterium astration factor contrary to the demands of many GCE models. To avoid any prejudice about deuterium dust depletion along different lines of sight that are used to determine the "true" D abundance, we propose a model-independent, statistical Bayesian method to address this issue and determine in a model-independent manner the undepleted ISM D abundance. We find the best estimate for the gas-phase ISM deuterium abundance to be $(\mathrm{D} / \mathrm{H})_{\text {ISM }} \geqslant(2.0 \pm 0.1) \times 10^{-5}$. Presented are the results of Prodanović et al. (2009).
\end{abstract}

Keywords. ISM: abundances, ISM: dust, Galaxy: abundances, Galaxy: evolution

\section{Introduction}

Deuterium is only created in significant amounts during the big bang nucleosynthesis (BBN) while all other processes destroy it (Epstein et al. 1976, Prodanović \& Fields 2003, Steigman 2007). Consequently, the deuterium abundance should monotonically decrease after the Big Bang, where the main destruction channel is the stellar processing. Because of this unique property deuterium can be used as a cosmic baryometer but also to discriminate between different Galactic chemical evolution (GCE) models (see eg. Steigman et al. 2007, Prodanović \& Fields 2008, Vangioni-Flam et al. 1994). Specifically, given that deuterium is mostly destroyed in stars, its abundance $\left(y_{\mathrm{D}} \equiv 10^{5}(\mathrm{D} / \mathrm{H})\right)$ can be used to probe the fraction of the interstellar gas that has never been processed through stars (Steigman \& Tosi 1992, 1995), which is often quantified with the astration factor $f_{\mathrm{D}} \equiv y_{\mathrm{DP}} / y_{\mathrm{D}, \mathrm{ISM}}$, the ratio of the primordial $\mathrm{D}$ abundance (the $\mathrm{D}$ to $\mathrm{H}$ ratio by number) to the ISM D abundance.

The primordial D abundance, $y_{\mathrm{DP}}$, predicted by the BBN model (Cyburt et al. 2003, Steigman 2007) based on the cosmic microwave background observations (Spergel et al. 


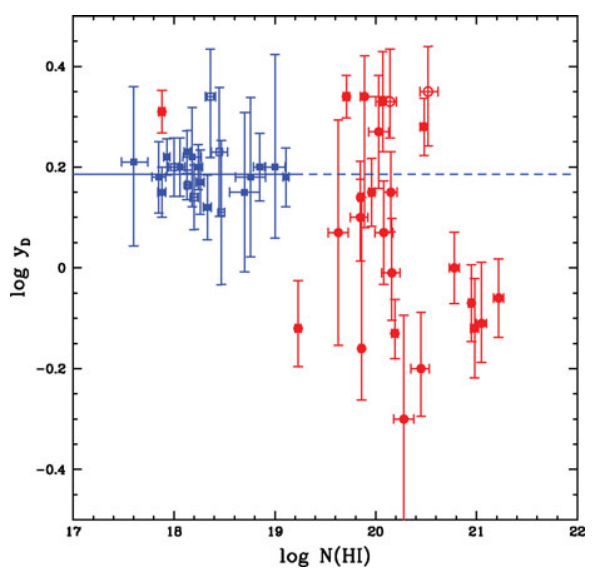

Figure 1. The logs of the deuterium abundances versus the logs of the $\mathrm{H}_{\mathrm{I}}$ column densities $\left[\mathrm{cm}^{-2}\right]$ for the 46 LOS from Table 2 of Linsky et al.(2006). The filled symbols are for the 38 LOS which have iron abundance data, while the open symbols are for the 8 LOS which lack iron abundances. The squares (blue) are for the LOS within the Local Bubble (LB) and the circles (red) are for the non-LB (nLB) LOS. The solid line is at the mean D abundance for the LB LOS $\left(\log y_{\mathrm{D}, \mathrm{LB}}=0.19\right)$; the dashed line is its extension to the nLB LOS.

2007), is in excellent agreement with D abundance observations at high-redshift systems (Pettini et al. 2008). However, observations of the gas-phase deuterium abundance in the interstellar medium (ISM) have revealed large variations over different lines of sight (LOS) by factors of $\sim 4\left(0.5 \lesssim y_{\mathrm{D}, \mathrm{ISM}} \equiv 10^{5}(\mathrm{D} / \mathrm{H})_{\mathrm{ISM}} \lesssim 2.2\right.$; Jenkins et al. 1999 , Hébrard et al. 2002, Sonneborn et al. 2000, Hoopes et al. 2003). The range of these variations is best seen on Figure 1, where we have plotted the logs of the gas-phase D/H $\left(\log y_{\mathrm{D}} \equiv 5+\log \mathrm{N}(\mathrm{D} \mathrm{I})-\log \mathrm{N}(\mathrm{H} \mathrm{r})\right)$ as a function of the logs of the $\mathrm{H}$ i column densities toward 46 different LOS inside and outside of the Local Bubble (LB) where the data has been taken from Linsky et al.(2006). Blue squares represent 21 LB LOS, while red circles represent 25 LOS from outside of the LB. Filled symbols represent those LOS for which iron abundance has also been measured. As an explanation for the observed scatter, it has been proposed that the gas-phase deuterium is depleted onto dust grains preferential to hydrogen (Jura 1982, Draine 2006). This hypothesis is in agreement with findings that the gas phase D/H correlates positively with refractory elements (Prochaska et al. 2005, Linsky et al. 2006, Steigman et al. 2007).

Arguing that deuterium is severely depleted onto dust grains, Linsky et al.(2006) have used the five highest $\mathrm{D} / \mathrm{H}$ ratios to determine the lower bound to the true, gas-phase ISM deuterium abundance $y_{\mathrm{D}, \mathrm{ISM}} \geqslant 2.31 \pm 0.24$, which is at the level of $\sim 80 \%$ of the primordial deuterium abundance $y_{\mathrm{DP}}=2.82_{-0.19}^{+0.20}$ (Pettini et al. 2008). Adopting this new estimate of the ISM D abundance, and the primordial abundance of Pettini et al.(2008), one finds the upper bound to the astration factor $f_{\mathrm{D}} \leqslant 1.22 \pm 0.15$, which is inconsistent with many GCE models that require $1.4 \lesssim f_{\mathrm{D}} \lesssim 1.8$ (Steigman et al. 2007 ). In order to account for such high ISM deuterium abundance and a low astration factor, most GCE models would require large infall rates of close to pristine material (Romano et al. 2006, Prodanović \& Fields 2008).

Because the observed variations and a possibly severe dust-depletion complicate determination of the true, gas-phase, deuterium abundance in the ISM, we have proposed a Bayesian statistical approach $(\S 2)$ in analyzing the deuterium data which yields the maximum likelihood estimate of the true, undepleted, gas-phase ISM deuterium abundance $y_{\mathrm{D}, \max }($ Prodanović et al. 2009). The underlying assumption of this analysis is that the 
observed spread in abundance measurements is the result of incompletely homogenized dust depletion. Our results are presented in $\S 3$, and conclusions in $\S 4$.

\section{Bayesian analysis}

To limit ourself to fewest assumptions possible, we have analyzed the FUSE ISM gasphase deuterium abundance measurements (46 LOS from Table 2. of Linsky et al. 2006) by using a statistical Bayesian approach, which was first developed by Hogan et al.(1997) for the purpose of determining the primordial helium abundance that best fits the observations. The main assumption of this approach is that, in our case, depletion of gas-phase deuterium abundance is present at some level, but where there is no further assumption about the nature, level or spatial distribution of this depletion. In this sense, the Bayesian statistical approach is model independent. Thus, the available data are analyzed in an unbiased, statistical manner, which as a result determines the two parameters - the undepleted (or a lower bound to it), gas-phase ISM D abundance $y_{\mathrm{D}, \mathrm{ISM}} \gtrsim y_{\mathrm{D}, \max }$, and the depletion parameter $w \equiv y_{\mathrm{D}, \max }-y_{\mathrm{D}, \min }$.

The values of the two parameters, $y_{\mathrm{D}, \max }$ and $w$, that best fit the set of measurements $y_{\mathrm{D}, i}$, with errors $\delta_{i}$, are found by determining the maximum value of the likelihood function

$$
\begin{aligned}
\mathscr{L}\left(y_{\mathrm{D}, \max } ; w\right) & =\prod_{i} P\left(y_{\mathrm{D}, i} \mid y_{\mathrm{D}, \text { max }} ; w\right) \\
& =\prod_{i} \int d y_{\mathrm{D}, i, \mathrm{~T}} P\left(y_{\mathrm{D}, i} \mid y_{\mathrm{D}, i, \mathrm{~T}}\right) P\left(y_{\mathrm{D}, i, \mathrm{~T}} \mid y_{\mathrm{D}, \max } ; w\right)
\end{aligned}
$$

The probability distribution $P\left(y_{\mathrm{D}, i} \mid y_{\mathrm{D}, i, \mathrm{~T}}\right)$ represents the probability of measuring the abundance $y_{\mathrm{D}, i}$ along the $i$ th LOS , given the true, error-free (but possibly depleted!) abundance $y_{\mathrm{D}, i, \mathrm{~T}}$ along that LOS. This distribution reflects the fact that real observed data have errors, and thus we assume that the shape of this distribution is a Gaussian of width $\sigma_{i}$. We treat the data with asymmetrical errors by assuming that $\sigma_{i+}$ corresponds to $y_{\mathrm{D}, i}>y_{\mathrm{D}, i, \mathrm{~T}}$ and $\sigma_{i-}$ to $y_{\mathrm{D}, i}<y_{\mathrm{D}, i, \mathrm{~T}}$. The probability distribution $P\left(y_{\mathrm{D}, i, \mathrm{~T}} \mid y_{\mathrm{D}, \max } ; w\right)$ is the probability of finding the true, depleted, LOS deuterium abundance $y_{\mathrm{D}, i, \mathrm{~T}}$, given the values of the maximum and minimum gas phase ISM deuterium abundances, $y_{\mathrm{D}, \max }$ and $y_{\mathrm{D}, \min }=y_{\mathrm{D}, \max }-w$ respectively. If we assume that depletion is due to dust, then $P\left(y_{\mathrm{D}, i, \mathrm{~T}} \mid y_{\mathrm{D}, \max } ; w\right)$ represents the distribution of dust depletion along different LOS $w_{i}$. Given that we know very little about this distribution, and to limit ourselves to the fewest assumptions possible, we adopt three simplest forms of this depletion distribution: a tophat (all levels of depletion are equally probable), a positive-bias (favors low depletion) and a negative-bias (favors large depletion) function. Functional forms of the three depletion distributions for $y_{\mathrm{D}, \min } \leqslant y_{\mathrm{D}, i, \mathrm{~T}} \leqslant y_{\mathrm{D}, \max }$ are given in Eq. (2.3), while outside of this range $P\left(y_{\mathrm{D}, i, \mathrm{~T}} \mid y_{\mathrm{D}, \max } ; w\right)=0$. All depletion distributions are normalized to unity when integrated over $y_{\mathrm{D}, i, \mathrm{~T}}$.

$$
P_{t}\left(y_{\mathrm{D}, i, \mathrm{~T}} \mid y_{\mathrm{D}, \text { max }} ; w\right)=\left\{\begin{array}{cc}
1 / w, & \text { Top-hat } \\
2\left(y_{\mathrm{D}, i, \mathrm{~T}}-y_{\mathrm{D}, \text { min }}\right) / w^{2}, & \text { Positive-bias } \\
2\left(y_{\mathrm{D}, \mathrm{max}}-y_{\mathrm{D}, i, \mathrm{~T}}\right) / w^{2}, & \text { Negative-bias }
\end{array}\right.
$$

With a choice of depletion distribution and a set of deuterium abundance measurements with errors $y_{\mathrm{D}, i}, \delta_{i}$, from Equation (2.2) one can numerically calculate the likelihood function $\mathscr{L}\left(y_{\mathrm{D}, \max } ; w\right)$ for a choice of parameter $\left(y_{\mathrm{D}, \max } ; w\right)$ values. Likelihood is then calculated for a range of parameter values, where the combination of values that results in 
the maximum likelihood determines the most likely value of undepleted, gas-phase ISM deuterium abundance $y_{\mathrm{D}, \max }$ and the corresponding depletion parameter $w$, that best fit the observed abundances (with their errors) with a spread that results from depletion of deuterium onto dust (Prodanović et al. 2009).

\section{Results}

Because the spread between the observed abundances is very different for only LB and only non-LB LOS (Fig. 1), we have first analyzed the two subsets of data separately. The analyzed data have been taken from Table 2 of Linsky et al.(2006), and there are $21 \mathrm{LB}$ LOS and 25 non-LB LOS (about LB and non-LB LOS assignment see Linsky et al. 2006, Prodanović et al. 2009).

Measured LB deuterium abundances show very little scatter, and thus it is not surprising that all three shapes of depletion distribution given in Eq. (2.3) yield almost the same maximum likelihood udepleted, gas-phase deuterium abundance $y_{\mathrm{D}, \max } \approx 1.5$ which is consistent with no depletion $w \approx 0$. The likelihood contours for the top-hat case and for the LB data are shown on the top panel of Fig. 2. This deuterium abundance that best fits the LB observations results in the astration factor $f_{\mathrm{D}} \leqslant 1.8$ (Prodanovic et al. 2009) which is consistent with a range of successful GCE models presented in Steigman et al.(2007). In terms of the GCE models presented in Prodanović \& Fields (2008) where the infall rate of the pristine material is taken to be proportional to the star formation rate with the proportionality constant $\alpha$, our new ISM deuterium abundance for this case, is consistent with low infall rates $\alpha \lesssim 0.1$ and return fractions of $R \lesssim 0.5$ (fraction of the stellar mass that is returned to the ISM), which can now accommodate even the more recent initial mass functions.

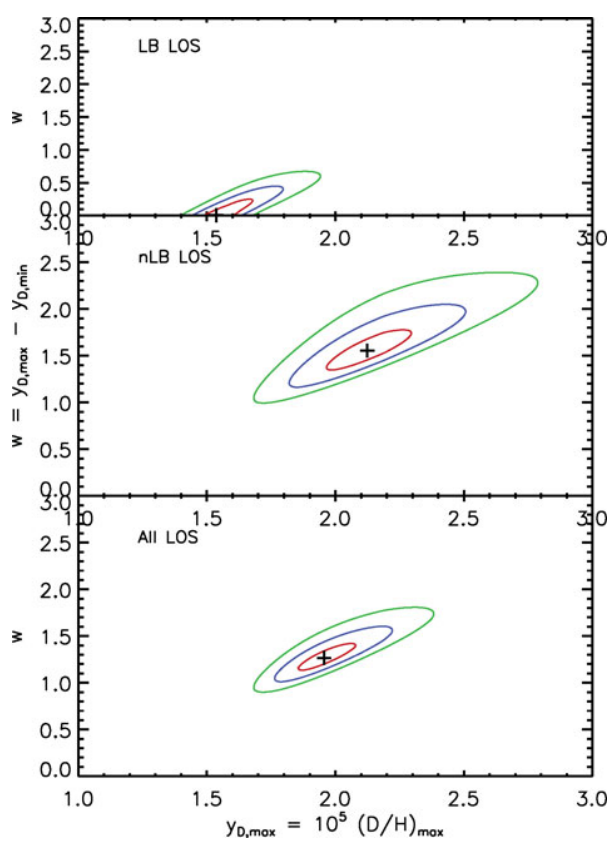

Figure 2. Likelihood contours $(68 \%, 95 \%, 99 \%)$ in the $w-y_{\mathrm{D}, \max }$ plane for the 21 LB LOS (top panel), the $25 \mathrm{nLB}$ LOS (middle panel), and all $46 \mathrm{LOS}$ (bottom panel), using the top-hat prior. 
On the other hand, observations of the gas-phase D abundances along different LOS outside of the LB, show significant scatter which can be seen from Fig. 1. The maximum likelihood values for the non-LB data set and the top-hat depletion distribution are $y_{\mathrm{D}, \max }=2.1$ and $w=1.6$. This result is presented on the middle panel of Figure 2 (Prodanović et al. 2009). The resulting astration factor $f_{\mathrm{D}, \mathrm{LB}} \leqslant 1.3$ is within the errors consistent with some GCE models discussed in Steigman et al.(2007). Within our new ISM D abundance in this case, a wide range of infall rates required by the GCE models of Prodanović \& Fields (2008) is now allowed with the infall parameter $0 \lesssim \alpha \lesssim 1$ where the corresponding return fractions can be found in the range are $0.2 \lesssim R \lesssim 0.4$, which can again accommodate even the modern initial mass functions.

Finally, when the full data set with all 46 LOS deuterium observations is considered with a choice of a top-hat depletion distribution, the maximum likelihood value of the undepleted, gas-phase deuterium abundance that best fits the observed data is found to be $y_{\mathrm{D}, \max }=2.0$ with a non-zero depletion of $w=1.3$. This result is presented on the bottom panel of Figure 2 (Prodanović et al. 2009). The corresponding astration factor is found to be $f_{\mathrm{D}} \leqslant 1.4$ which is consistent with some but not all GCE models adopted Steigman et al.(2007). Similarly to the non-LB case, a wide range of the GCE models presented in Prodanović \& Fields (2008) is allowed with our new estimate of the undepleted, gas-phase D abundance.

The two subsets of data, only LB and only non-LB, as well as the complete set of all 46 LOS measurements, have all been analyzed with all three depletion probability distributions from Eq. (2.3). In all cases, it was found that the top-hat distribution results in the largest maximum likelihood value, i.e. that it fits the spread observed in the data the best (Prodanović et al. 2009).

\section{Conclusion}

Observations of deuterium in the ISM have revealed large variations (Jenkins et al. 1999, Hébrard et al. 2002, Sonneborn et al. 2000, Hoopes et al. 2003) which complicates the determination of the unique, ISM deuterium abundance (see Figure 1). It was proposed that the observed variations originate from inhomogeneous depletion of deuterium onto dust grains preferential to hydrogen (Jura 1982, Draine 2006). In the light of this hypothesis, Linsky et al.(2006) have proposed that most of the deuterium in the ISM has been depleted and that the true, undepleted $\mathrm{D}$ abundance is $y_{\mathrm{D}, \mathrm{ISM}} \geqslant 2.31 \pm 0.24$ which is at the $\sim 80 \%$ level of the primordial D abundance (Pettini et al. 2008). If the ISM D abundance is indeed so high this in turn creates tension with otherwise successful GCE modes (see eg. Romano et al. 2006, Prodanović \& Fields 2008).

Because of the importance of knowing the undepleted, gas-phase ISM D abundance, we have employed a model-independent Bayesian statistical analysis first used by Hogan et al.(1997) to determine the primordial helium abundance. By determining the maximum likelihood value of the likelihood function for the assumed depletion distribution function, this approach yields the values of two parameters, undepleted, gas-phase deuterium abundance $y_{\mathrm{D}, \max }$ and the depletion parameter $w$, that best fit the observed data and scatter which is assumed to be due to different, in our case dust, depletion levels. We have investigated three different depletion distribution functions: a top-hat that equally favors both high and low depletion levels, a positive-bias that favors low-depletion and a negative-bias function that favors large depletion. Of the three depletion distributions the uniform top-hat distribution results in the largest maximum likelihood and thus fits the data best. 
We have applied the Bayesian statistical analysis to the data set of deuterium abundance measurements along 46 different LOS, where the data has been adopted from Linsky et al.(2006). Figure 2 shows likelihood contours for the top-hat depletion distribution and for Bayesian analysis applied to 3 data subsets: LB only, non-LB only and the full data set. We found that the LB deuterium abundance measurements are consistent with having zero depletion $w=0$ and $\mathrm{D}$ abundance of $y_{\mathrm{D}, \mathrm{LB}}=1.5(1 \pm 0.03)$. Unlike the LB, the non-LB data require significant depletion. Because it is still unclear whether the LB D is undepleted while some or all non-LB LOS have been enriched by unmixed infall, or if LB is uniformly depleted, we find that the best estimate of the undepleted, gas-phase ISM deuterium abundance is that which follows from analyzing the full 46 LOS data set, with a top-hat depletion distribution function, and is $y_{\mathrm{D}, \mathrm{ISM}} \geqslant y_{\mathrm{D}, \max }=2.0 \pm 0.1=2.0(1 \pm 0.05)$ (Prodanović et al. 2009). With the primordial deuterium abundance adopted from Pettini et al.(2008), we find that our new ISM D abundance corresponds to the astration factor $f_{\mathrm{D}} \leqslant 1.4 \pm 0.1$ that is consistent with some successful GCE models (Steigman et al. 2007, Prodanović \& Fields 2009), which releases some tension with GCE models that is created when a high ISM deuterium abundance, such as that of Linsky et al.(2006), is required.

\section{Acknowledgements}

The work of TP is supported in part by the Provincial Secretariat for Science and Technological Development, and by the Ministry of Science of the Republic of Serbia under project number 141002B.

\section{References}

Cyburt, R. H., Fields, B. D., \& Olive, K. A. 2003, Phys. Lett. B, 567, 227

Draine, B. T. 2006, Astrophysics in the Far Ultraviolet: Five Years of Discovery with FUSE, 348,58

Epstein, R. I., Lattimer, J. M., \& Schramm, D. N. 1976, Nature, 263, 198

Hébrard, G., et al. 2002, ApJS, 140, 103

Hogan, C. J., Olive, K. A., \& Scully, S. T. 1997, ApJL, 489, L119

Hoopes, C. G., Sembach, K. R., Hébrard, G., Moos, H. W., \& Knauth D. C. 2003, ApJ, 586, 1094

Jenkins, E. B., Tripp, T. M., Woźniak, P. R., Sofia, U. J., \& Sonneborn, G. 1999, ApJ, 520, 182

Jura, M. 1982, Advances in Ultraviolet Astronomy, Kondo, Y., Mead, J., Chapman, R. D., eds., NASA, Washington, p. 54

Linsky, J. L., et al. 2006, ApJ, 647, 1106

Pettini, M., Zych, B. J., Murphy, M. T., Lewis, A., \& Steidel, C. C. 2008, MNRAS, 391, 1499

Prochaska, J. X., Tripp, T. M., \& Howk, J. C. 2005, ApJ, 620, L39

Prodanović, T. \& Fields, B. D. 2003, ApJ, 597, 48

Prodanović, T. \& Fields, B. D. 2008, JCAP, 9, 3

Prodanović, T., Steigman, G., \& Fields, B. D. 2009, submitted to MNRAS, arXiv:0910.4961

Romano, D., Tosi, M., Chiappini, C., \& Matteucci, F. 2006, MNRAS, 369, 295

Sonneborn, G., Tripp, T. M., Ferlet, R., Jenkins, E. B., Sofia, U. J., Vidal-Madjar, A., \& Woźniak, P. R. 2000, ApJ, 545, 277

Spergel, D. N., et al. 2007, ApJS, 170, 377

Steigman, G. \& Tosi, M. 1992, ApJ, 401, 150

Steigman, G. \& Tosi, M. 1995, ApJ, 453, 173

Steigman, G., Romano, D., \& Tosi, M. 2007, MNRAS, 378, 576

Steigman, G. 2007, Ann. Rev. Nucl. Part. Sci, 57, 463

Vangioni-Flam, E., Olive, K. A., \& Prantzos, N. 1994, ApJ, 427, 618 